\title{
Beweglich bleiben!
}

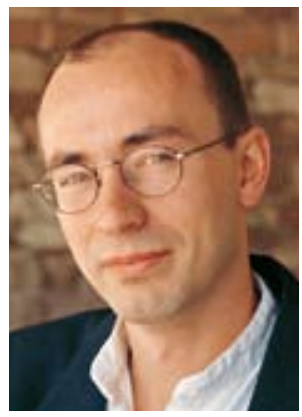

Dr. med. Dieter Schmid, Redaktionsleitung

\section{Quellen:}

[1] www.healthy-campus.de [2] Die Entwicklung der Studienabbruchquote (...). HIS: Projektbericht, Februar 2008 [3] Via medici-PJ-Umfrage 2011; www.thieme.de] 2011; www.thieme.de/
viamedici/medizinstudium/ pj/umfrage_11.html
Schon gewusst? Jeder vierte Student ist ein „Couch-Potatoe“. Laut einer Untersuchung der Deutschen Sporthochschule Köln betreiben 25,4\% aller Erstsemester keinen Sport zumindest in Bonn, wo die Studie durchgeführt wurde [1]. Gilt das auch für Sie? Sicher nicht! Schließlich lesen Sie Via medici - und studieren damit höchstwahrscheinlich Medizin. Und in diesem Fach gilt es beweglich zu bleiben. Zumindest im übertragenen Sinn - denn derzeit ändern sich gewaltig die Studienperspektiven ...

Was war das in den letzten Monaten für ein Gezerre! Zuerst durften sich junge Mediziner über eine sehr schöne Nachricht freuen: Das monströse Hammerexamen sollte endlich in zwei Teile aufgespalten werden - in eines vor und eines nach dem PJ. Wenige Wochen nach Bekanntgabe dieses Plans dann die böse Überraschung: Am 15. Februar zauberten die Landesgesundheitsminister den Vorschlag aus der Tasche, dass man im Zuge der Überarbeitung der Approbationsordnung (AO) ja gleich auch das Problem des Hausärztemangels lösen könne - und zwar, indem man das bei den Studenten beliebte Wahltertial abschafft und dafür ein Pflichttertial Allgemeinmedizin einführt. Das hätte zur Folge gehabt, dass man große Fächer neben der Inneren und der Chirurgie, wie die Pädiatrie (unser Titelthema auf S. 18), innerhalb des Studiums nicht mehr vernünftig kennenlernen hätte können. Entsprechend entfachte dieser Vorschlag einen Sturm der Entrüstung. Die Deutsche Gesellschaft für Allgemeinmedizin legte daraufhin das Ei des Kolumbus auf den Tisch: das zusätzliche Pflicht-QUARTAL Allgemeinmedizin. Kleiner Pferdefuß an dieser Lösung: Die Tertiale der Chirurgen und Internisten wären um einen Monat gekappt worden - was wiederum deren Verbände auf die Barrikaden führte. Am 11. Mai hat der Bundesrat das monatelange Geschacher endlich beendet. Die neue AO kommt - mit gesplittetem Hammerexamen und ohne Zwangstertial bzw. -quartal in der Allgemeinmedizin!

Und der Bundesrat macht den Medizinstudenten noch ein Geschenk: Ab 2013 soll jeder PJler prinzipiell an jedem deutschen Lehrkrankenhaus sein Praktisches Jahr absolvieren können. Damit wird ein Traum wahr! Als Hamburger wird man so seine PJ-Tertiale nicht nur in Bern, Boston oder Bangalore absolvieren können, sondern auch in so „exotischen“ Orten wie Stuttgart, Greifswald oder München! Deshalb meine Empfehlung: Bleiben Sie beweglich - und nutzen Sie dieses Angebot! Wie unsere aktuelle PJ-Umfrage zeigt, hat sich im Bereich der Ausbildung im PJ zwar bereits einiges zum Positiven verändert (S. 16). Aber noch ist Luft nach oben. Und wenn sich die Kliniken ein bisschen Mühe geben müssen, um Nachwuchsärzte anzulocken, kann das der Qualität des Praktischen Jahres nur guttun ...

Ein erfolgreiches Restsemester wünscht

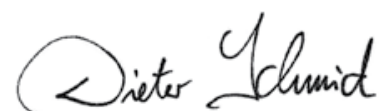

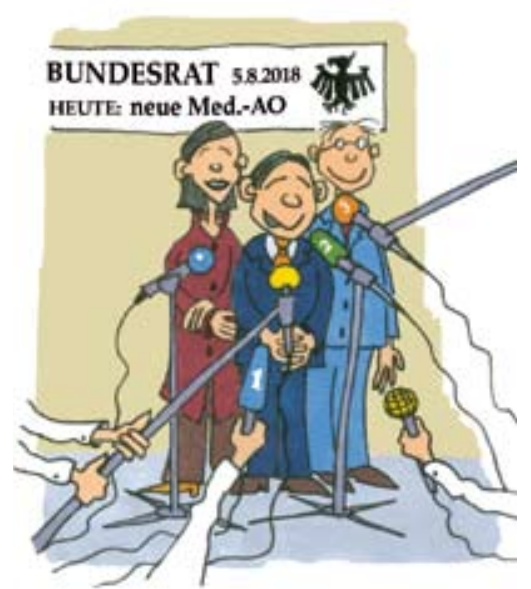

„... darf ich folgenden Kompromiss verkünden: Das PJ besteht künftig aus 21 Tertialen

à 1,3 Monaten, in denen jedes Fachgebiet verpflichtend durchlaufen wird ..."

ONLINE-UMFRAGE

\section{Bereuen Sie Ihre Studienwahl?}

Die meisten Medizinstudenten starten hoch motiviert in ihr Studium. Davon zeugt auch die im Vergleich zu anderen Studiengängen extrem niedrige Abbrecherquote [2]. In der Medizin bricht einer von zwanzig sein Studium ab, in den Technischen Wissenschaften ist es zum Beispiel einer von vier! Und doch: Auf dem Weg zum Traumberuf scheint vielen Jungmedizinern die Begeisterung abhanden zu kommen. Laut unserer PJ-Umfrage möchten 5\% nach dem Studium in einem komplett anderen Bereich arbeiten. 9\% möchten zwar im medizinischen Bereich bleiben, aber nicht als „Weißkittel“: Sie erwägen eine Stelle in der Industrie, einem Fachverlag oder in der Beratung [3]. Es sieht so aus, als ob so mancher Studienabsolvent das Rad der Zeit gerne zurückdrehen würde ... Wie sieht das bei Ihnen aus?

Angenommen Sie hätten die Wahl: Würden Sie nochmals mit dem Medizinstudium beginnen?

Mitmachen unter: www.thieme. de/viamedici/aktuelles/aktion/ miniumfrage3-12.html
- Ergebnis der Umfrage in Via medici 2.12: Wir fragten, was Sie von einem Pflichttertial Allgemeinmedizin halten. Eindeutige 96\% sprachen sich gegen diese Idee aus. Nur $4 \%$ fanden das sinnvoll. „Warum will man uns die Möglichkeit nehmen, das zu lernen, was uns begeistert?“, fragen sich die Gegner. Sie sind überzeugt, dass die Allgemeinmedizin mit anderen Mitteln um Nachwuchs werben müsse. Befürworter finden das Pflichttertial wichtig, um sich allgemeinmedizinische Kenntnisse anzueignen und dem Patienten einmal „auf Augenhöhe“ zu begegnen. Kommentare unter: .../aktuelles/aktion/miniumfrage2-12.html 